
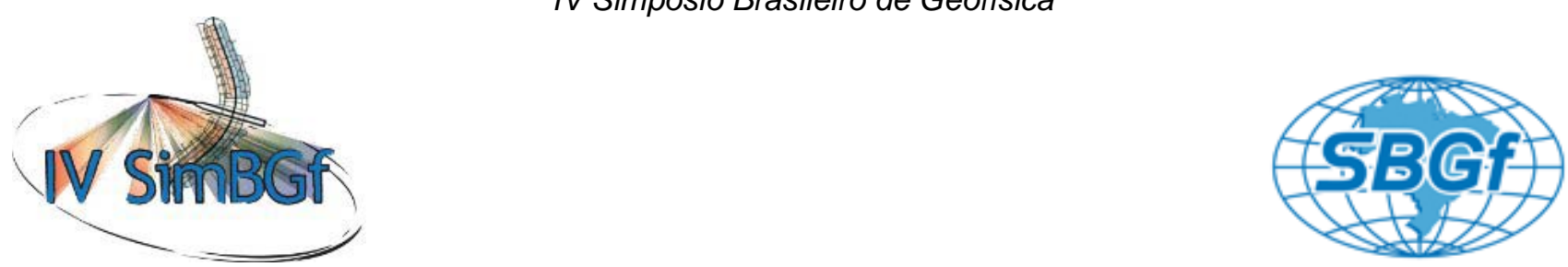

\title{
DETERMINAÇÃO DO DIÂMETRO DE INVASÃO DO FLUIDO DE PERFURAÇÃO EM RESERVATÓRIOS CARBONÁTICOS ATRAVÉS DA GEOFÍSICA EM POÇO
}

\author{
Mariléa G.S. Ribeiro, Alfredo M.V. Carrasco \& A. Abel G. Carrasquilla, UENF/CCT/LENEP - Macaé, RJ
}

Copyright 2010, SBGf - Sociedade Brasileira de Geofísica

Este texto foi preparado para a apresentação no IV Simpósio Brasileiro de Geofísica, Brasília, 14 a 17 de novembro de 2010. Seu conteúdo foi revisado pelo Comitê Técnico do IV SimBGf, mas não necessariamente representa a opinião da SBGf ou de seus associados. É proibida a reprodução total ou parcial deste material para propósitos comerciais sem prévia autorização da SBGf.

\section{Resumo}

Neste trabalho, estudamos, de forma uni e tridimensional, o processo de invasão do fluido de perfuração num reservatório carbonático, através dos valores das resistividades medidas pelo perfil de indução eletromagnética. As propriedades físicas e químicas desse fluido controlam a operação de perfuração de poços, possibilitando a sua execução em ambientes com diferentes características geológicas. Durante esse processo ocorre a perda do filtrado da lama de perfuração para a formação, quando partículas em suspensão tamponam os poros na parede do poço e formam o reboco na parede do poço. Isto provoca um processo de invasão nas vizinhanças dos poços, causado pela diferença de pressões nas zonas adjacentes ao poço. Como conseqüência disto, uma camada contendo hidrocarbonetos mostra os perfis de resistividade com três zonas com diferentes valores de resistividades: a lavada, a de transição e a virgem. Entretanto, toda ênfase é dada à medição da resistividade da zona virgem, pois é aquela que está diretamente ligada à estimativa da saturação de óleo. Para tanto, se utilizam aquelas ferramentas de indução que medem valores profundos, como a 6FF40. No nosso trabalho, os resultados da simulação unidimensional de três camadas num modelo de um meio geológico similar ao ambiente do pré-sal, mostra um perfil de invasão de até $1,2 \mathrm{~m}$, mas com pouco contraste de resistividade entre a camada de sal e o reservatório carbonático. A modelagem tridimensional, por outro lado, mostra que não é possível diferenciar a camada de sal do reservatório com os parâmetros assumidos na simulação.

\section{Introdução}

O processo de perfuração de um poço precisa manter as paredes do poço estável, seja para prevenir a invasão de fluidos ao poço ou a queda das paredes para formar cavernas. Para isto, é preciso que se tenha um diferencial de pressão entre o poço e a formação, o que vai depender muito do peso do fluido. O raio de invasão da lama de perfuração $\left(R_{\mathrm{i}}\right)$, por outro lado, é uma variável que depende da pressão hidrostática do fluido de perfuração e das propriedades petrofísicas das rochas, tais como a porosidade, permeabilidade, quantidade e variedade de minerais, pressão capilar, etc. (Serra, 2007). Ribeiro \& Carrasquilla (2008), mostraram um modelo de invasão unidimensional do filtrado focado em reservatórios siliciclásticos, onde o processo deposicional apresentava uma petrofísica bem complexa, quanto a porosidade, permeabilidade, fluido molhante, interconectividade dos poros e um contexto complexo de minerais, tais como quartzo, ilita, montmorilonita, dolomita, calcita e outros, que interferiam diretamente no processo de invasão do fluido de perfuração de poços de petróleo.

Dentro deste contexto, os reservatórios carbonáticos também apresentam uma ampla heterogeneidade geológica e petrofísica (Lucia, 2004). Nos carbonatos a porosidade e a permeabilidade possuem pouca correlação espacial e são extremamente variáveis em escalas de pés ou polegadas, e a permeabilidade apresenta uma distribuição quase aleatória (Bovan et al., 2003). Ainda segundo esses autores, os carbonatos apresentam uma porosidade inter-partícula e vários tipos de porosidade tipo "vuggy" e suas principais estruturas rochosas são da forma "grainstone" e "packstones", com uma estrutura lamosa dominante. Alguns cristais aparecem na estrutura lamosa como se fosse um controle do tamanho de poro, que pode ser modificado pelo processo da diagênese. Se a formação é permeável e existe presença de invasão, o mesmo é governado principalmente pela pressão hidrostática da lama de perfuração e pelas características do reboco. Assim, quanto menor a porosidade, maior será a invasão dentro da formação. Na medida que a maioria dos carbonatos apresentarem porosidade bastante baixa, geralmente serão encontrados invasões de moderadas a profundas (Chilingar et al, 1972)

Davis (1979) apresentou um estudo que demonstrou que a pirita está presente nos carbonatos como cristais nas fraturas, preenchendo totalmente ou parcialmente o espaço poroso, até os "vuggy" do calcário ou calcário dolomitizado. Em análise das características de um reservatório carbonático em termos de conteúdo, magnitude de fluido no espaço poroso e a geometria do ambiente, ele mostra ter de $1-5 \%$ de pirita, podendo ocorrer quantidades menores que $1 \%$ e se ocorrer a condição de $0 \%$, ele afirma ser uma condição de cuidado na análise. Ele afirma ainda que a presença da pirita adicionada ao efeito de fratura ou porosidade vulgular contendo gás, e a presença de anidrita é uma situação de grande cuidado para os analistas de perfis de poços. 
Ramakrishnan et al. (2000) mostraram no seu estudo que a maior parte das interpretações petrofísicas são realizadas para reservatórios siliciclásticos, apesar de 50\% das reservas mundiais serem reservatórios carbonáticos. Dizeram ainda que a interpretação petrofísica dos reservatórios carbonáticos precisa ser minuciosa, devido a complexidades da sua estrutura porosa, que podem ser distribuídos de dois ou três modos diferentes (interpartícula, intra-partícula e "vug"), variando de acordo com a forma e o tamanho dos poros. O processo diagenético, ainda, tem muito a contribuir com a esta complexidade, pois ocorre nos carbonatos por dissolução química, reprecipitação, dolomitização, esforços mecânicos entre outros processos; e ainda existe a presença comum da anidrita.

\section{Metodologia}

Para realizar este trabalho, foi utilizado o modelo desenvolvido por Ribeiro \& Carrasquilla (2008), onde algumas adequações foram incorporadas para adaptar-se às novas características litológicas para reservatórios carbonáticos. A invasão é quantificada e registrada como mais um perfil de acordo com programa desenvolvido nesse trabalho na plataforma computacional MATLAB (2010).

Utilizando o mesmo modelo de três camadas, passamos agora a ter uma camada superior de $20 \mathrm{~m}$ de sal, uma camada intermediária de $20 \mathrm{~m}$ de reservatório carbonático e uma camada inferior de $20 \mathrm{~m}$ de folhelho (Figura 1). Tomamos como base essa litológia baseados na descoberta dos reservatórios do pré-sal na Bacia de Santos, os quais se localizam em águas ultra-profundas com 2.200 m de lâmina d'água, distantes $290 \mathrm{~km}$ da costa brasileira e localizados abaixo de uma camada de $2.000 \mathrm{~m}$ de sal (Almeida et al., 2010). A linha sísmica e a seção geológica (Figuras $2 \mathrm{a}$ e $2 \mathrm{~b}$ ) desta bacia foram retiradas da apresentação de Formigli (2009), baseada no trabalho de Moriak et al. (2008).

Cada camada apresenta certo grupo de minerais. Estes minerais são incluídos na litologia em percentuais, e ainda, incluímos os percentuais de cada fluido presente ou não em cada camada. Todavia variamos estes percentuais e verificamos como alguns destes minerais podem influenciar nos resultados da invasão, na respostas dos perfis e no efeito "annulus" (Figuras 3a e 3b), o que irá influenciar diretamente a saturação de fluidos no meio rochoso. Para a camada de sal, montamos uma composição baseada nos seguintes minerais: halita, silvita, gipsita, carnalita e anidrita. Já na camada de carbonato, incluímos pirita, calcopirita, dolomita, anidrita e carbonato de cálcio. E, por fim, na camada de folhelho, distribuímos os minerais tipo quartizo, calcita, montmorilonita, ilita e dolomita. Todos estes minerais formadores de rochas foram apresentados pela literatura como presentes em cada litologia (). No folhelho, entretanto, assumimos que a invasão é zero, quanto que no sal não ocorre invasão devido suas propriedades elásticas que a mesma apresenta em profundidade. Nos carbonatos, contudo, ocorre invasão baseada na índice de saturação de fluidos. Toda a composição mineral que utilizados em nosso trabalho, pertencentes a cada camada, aparecem mostrados na Figura 3.

Na segunda parte deste trabalho, foi realizada a modelagem tridimensional do perfil de indução profunda (6FF40), utilizando programa desenvolvido na linguagem FORTRAN no trabalho de Carrasco (2002). O modelo tridimensional assume uma distribuição radial de resistividades mostrada na Figura 5, que simula as diferentes zonas do processo de invasão nas vizinhanças do poço. Como se trata de um perfil de indução, nesse modelo, assumimos que o poço foi perfurado com lama base óleo com uma resistividade de $50 \mathrm{ohm}-\mathrm{m}$. As lamas base óleo têm um porção do seu conteúdo de água, que em contacto com a formação salina, incrementa a salinidade do filtrado de lama, além de acrescentar as condições de pressões e temperatura na seção de interesse. A parte aquosa, por ser menos viscosa, penetra com maior facilidade dentro da formação, e por ser um reservatório carbonático geralmente com baixa permeabilidade, aconteceria uma invasão de moderada a profunda. Assim os seguintes dados de resistividades foram considerados na simulação: zona lavada de raio de 0.87 m com 100 ohm$\mathrm{m}$, zona de transição de $1.3 \mathrm{~m}$ de raio com uma resistividade de $10^{3.7}$ ohm-m e finalmente a zona virgem com uma resistividade de $10^{5.7}$ ohm-m, segundo o esquema representado na figura 4 . A dimensão de cada cúbica na simulação é considerada com um valor de 0,216 m.

\section{Resultados}

A Figura 4 mostra o resultado da simulação unidimensional obtida para o processo de invasão do reservatório carbonético das Figuras 1 e 3 . Nessa Figura 4 podemos observar que no perfil de raios gama - GR (primeira pista), o sal apresenta valores altos, quanto que nos carbonatos os valores desse perfil são baixos. No folhelho, entretanto, os valores do perfil GR são altos, em acordo com a sua composição mineralógica assumida. No caso do perfil resistividade medido pela ferramenta de indução, obtivemos valores altos no sal, o que é uma resposta perfeitamente aceitável já que o sal nestas condições não contém água; quanto que no folhelho, os valores são baixos, ainda considerando uma saturação de fluido baixa para esta camada. No reservatório carbonático, porém, as resistividades apresentam valores significativos devido à presença de fluidos resistivos saturando o meio (óleo), podendo-se observar claramente a leitura da resistividade profunda com um valor alto $\left(\approx 10^{6}\right)$, pois neste teste consideramos um percentual de $25 \%$ de hidrocarboneto presente na rocha. Entretanto, o contraste de resistividade entre a camada de sal e o reservatório carbonático é alto (de mais de 200.000 ohm.m), o que não é evidente no gráfico devido à escala logarítmica.

Nos resultados da simulação tridimensional, considerando a distribuição de resistividades apresentadas na Figura 5, observamos, na Figura 6, que a resposta tridimensional do perfil de indução 6FF40 (linha azul) fica fortemente influenciada pelas altas resistividades do meio encaixante unidimensional plotada na mesma figura (linha 
preta), Observamos que a simulação tridimensional não mostra diferenças entre a camada de sal e o reservatório carbonático com os parâmetros de simulação adotados.

\section{Conclusões}

Neste trabalho observamos, na simulação unidimensional, que existe uma invasão profunda de até $1,2 \mathrm{~m}$ no reservatório carbonático modelado. Esse valor elevado pode ter origem na complexa estrutura porosa dos carbonatos, a qual é uma função do processo deposicional e diagenético, além do efeito natural que o filtrado exerce sobre os carbonatos. Os altos valores de resistividade na camada de sal e no reservatório carbonático atribuem-se, entretanto, aos altos valores assumidos na simulação para a composição mineralógica dessas camadas. Apesar do gráfico em escala logarítmica não mostrar, existe um alto contraste de resistividade entre o sal e os carbonatos. No caso da modelagem tridimensional, observamos que os resultados da perfilagem de indução da ferramenta 6FF40 são fortemente influenciados pelo meio encaixante estratificado, não sendo observado contraste entre a camada de sal e o reservatório carbonático com os parâmetros utilizados. Finalmente, mais simulações são necessárias para entender melhor o processo de invasão nos reservatórios carbonáticos, pois como assinalam vários autores, a cultura da perfilagem de poços é baseada em reservatórios siliciclásticos, precisando, portanto, uma adaptação para um melhor entendimento do que está acontecendo.

\section{Agradecimentos}

À Universidade Estadual Norte Fluminense Darcy Ribeiro (UENF) pela sua infraestrutura e à PETROBRÁS pelo apoio financeiro através de projeto de pesquisa na Rede CARMOD.

\section{Referencias}

Almeida, A.S., Lima, S.T.C., Rocha, P.S., Andrade, A. M. T., Branco, C.C.M, \& Pinto, A.C.C., 2010. CCGS Oportunities in the Santos Basin Pre-salt Developement, Petrobras, SPE 126566.

Bovan, K.G., Torres-Verdín, C., Mojde, D., Sigal, R., Zouioueche, F. \& Anderson, B. A, 2003. Case study integrating the physics of mud-filtrate invasion with the physics of induction logging: assessment of in-situ hydrocarbon saturation in the presence of deep invasion and highly saline connate water, University of Texas.

Carrasco, A.M.V., 2004. Simulação numérica tridimencional de perfis indutivos em poços tese doutorado, LENEP/UENF, Macaé-RJ, 87p

Chilingar, G., Mannon, R. \& Rieke H., 1972. Oil and Gas Production from Carbonate Rocks, Elsevier Publishing Company.

Davis D. G., 1979. Pyrite in Carbonate in Rocks - A Computer Technique. SPWLA Twentieth Annual Logging Symposium, June 2-6.
Formigli, J., 2009. Apresentação E\&P/PRESAL, PETROBRAS.

Lucia, F.J. 2004. Carbonate Reservoir Characterization An Integrated Approach, $2^{\text {nd }}$ edition, 336p.

MATLAB, 2010. User's manual.

Mohriak, W., Szartmari, P. \& Anjos, S.M.C., 2008. Sal Geologia e Tectônica Exemplos na Bacia de Campos, 448p.

Ribeiro, M.G.S. \& Carrasquilla A.A.G., 2008. Calculating mud drill invasion in theoretical reservoirs through geophysical well logs, $11^{\text {th }}$ International Congress of the Brazilian Geophysical Society.

Serra, O., 2007. Well logging and Reservoir Evolution, 250p.

Ramakrishnan T. S., Ramamoorthy R., Saito N., Flaum C., 2000. Method for interpreting carbonate reservoirs. USA. patent.

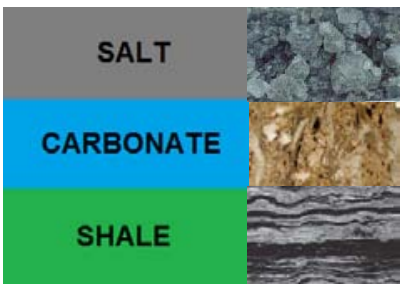

Figura 1. Modelo de três camadas 


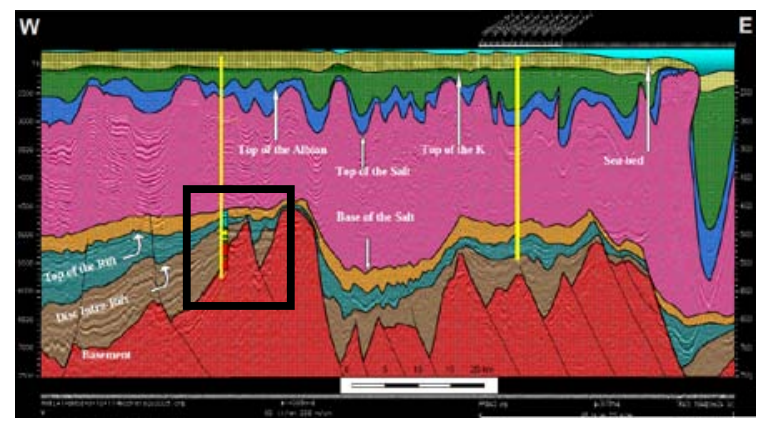

Figura 2a. Linha símica/seção geológica (Formigli, 2009).

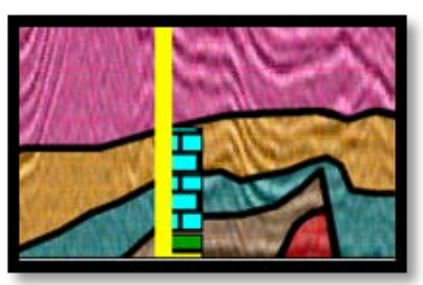

Figura 2b. Seção geológica (Formigli, 2009).

\begin{tabular}{|c|c|c|c|}
\hline \multirow{4}{*}{$\begin{array}{c}\text { composição mineral } \\
\text { trabalhada }\end{array}$} & sal & carbonato & folhelho \\
\cline { 2 - 4 } & halita & pirita & quartzo \\
\cline { 2 - 4 } & anidrita & calcopirita & calsita \\
\cline { 2 - 4 } & silvita & micrita & dolomita \\
\cline { 2 - 4 } & barrilha & dolomita & ilita \\
\cline { 2 - 4 } & gipsita hidratada & mica & caolinita \\
\cline { 2 - 4 } & bischofrita & feldaspato & anidrita \\
\hline
\end{tabular}

Figura 3. Composição mineral de cada camada na simulação unidimensional.
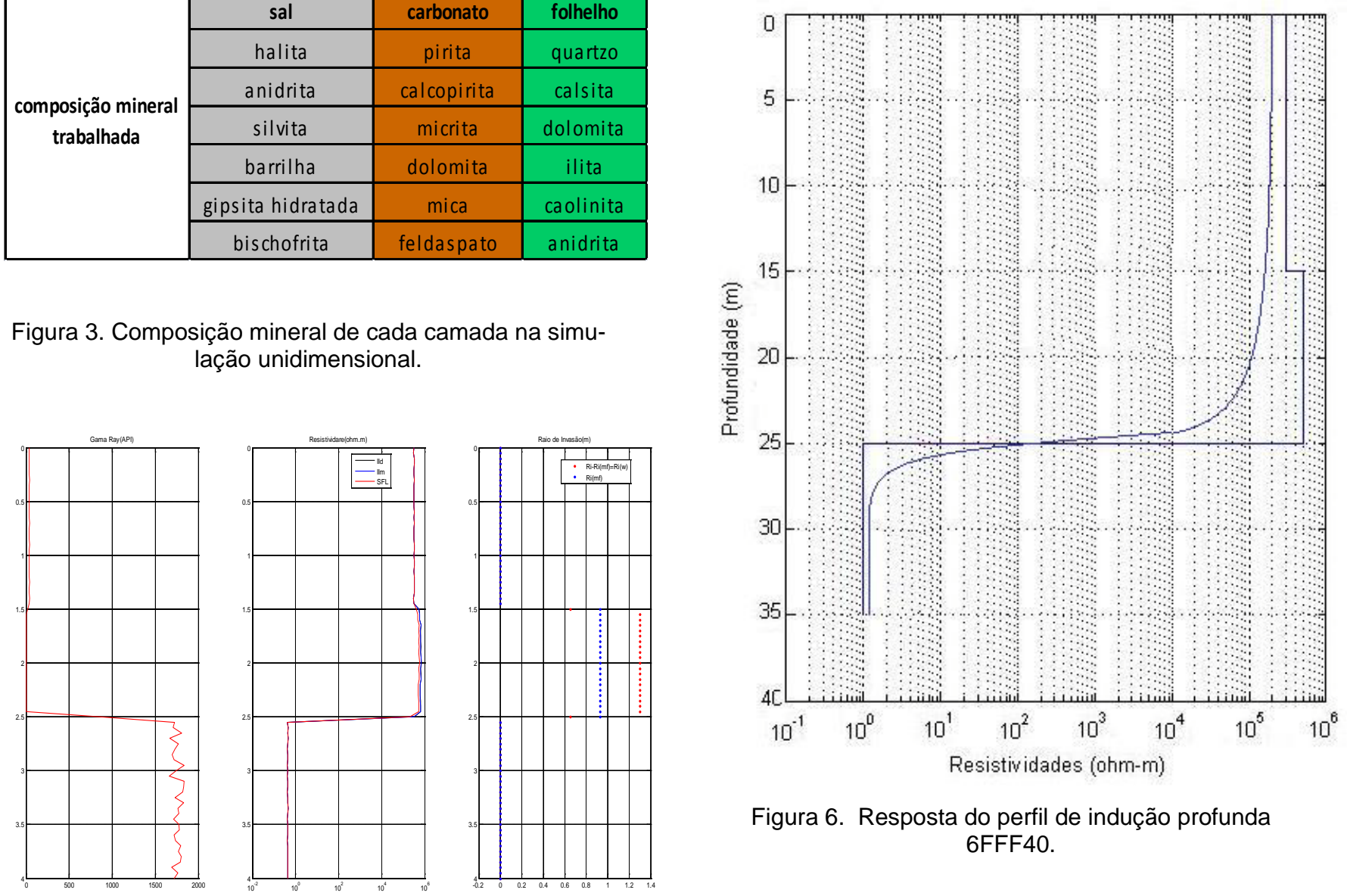

Figura 6. Resposta do perfil de indução profunda 6FFF40.

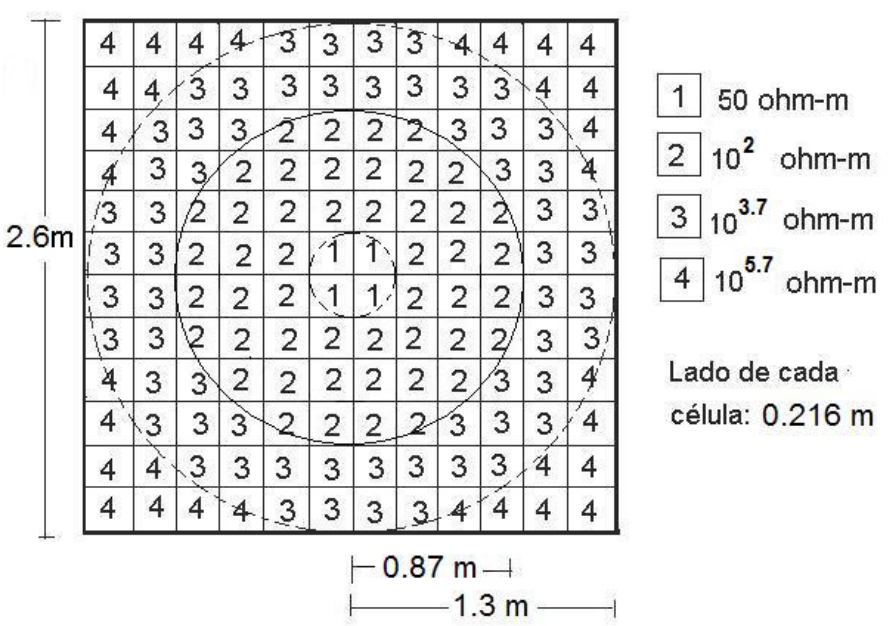

Figura 5 Distribuição radial de resistividades

Figure 4. Resultados da simulação unidimensional. 